\title{
Spectral analysis of voltages and currents during different modes of ferroresonance in switchgear
}

\author{
Zaipatimah $\mathrm{Ali}^{\mathrm{a}, \mathrm{b}^{*}}$, Vladimir Terzija ${ }^{\mathrm{b}}$ \\ ${ }^{a}$ Universiti Tenaga Nasional, Jalan IKRAM UNITEN, Kajang, 43000 Selangor, Malaysia \\ ${ }^{b}$ The University of Manchester, Sackville Street, Manchester, M13 9PL, United Kingdom
}

\begin{abstract}
This paper presents a spectral analysis of three different modes of ferroresonance. Voltage and current signals of the fundamental, subharmonic and chaotic ferroresonance modes are processed. The signals are obtained by modeling a ferroresonance circuit in alternative transient program-electro magnetic transient program (ATP-EMTP). The magnitude spectrum and total harmonic distortion are calculated for each case. The results show that voltages and currents of different modes of ferroresonance produce different features, which may be used for ferroresonance identification, classification and mitigation in the future.
\end{abstract}

Keywords: Feature extraction, ferroresonance, spectral analysis, total harmonic distortion

\section{Introduction}

Ferroresonance is a complex electrical phenomenon that is due to the interaction between the nonlinear characteristics of the transformer core and system's capacitance. It is a nonlinear phenomenon that is initiated by changes in the network topology; such as, switching operations or faults. The following are examples of electrical power system configurations that are prone to ferroresonance: a voltage transformer energized through the grading capacitance of one or more open circuit breakers, voltage transformer connected to an isolated neutral system and transformer that are accidentally energized in only one or two phases. More examples with detailed discussions are available in [1], [2]. Ferroresonance may cause overvoltages and overcurrents in an electrical power system. If the system is under sustained ferroresonance for a long time, it can cause dielectric and thermal problems in transmission and distribution systems. Ferracci has classified ferroresonance into four modes; namely, fundamental, subharmonic, quasi-periodic and chaotic [1]. The research areas related to ferroresonance range from modeling of the actual ferroresonance circuit in transient programs [3], [4] modeling the components (transformer modeling) inside the ferroresonance circuit [5], [6], understanding the behaviour of ferroresonance using chaos theory and nonlinear dynamics [7], [8], and the identification, detection and classification of ferroresonance signals from other transient disturbances [9].

Despite the large number of studies that have been conducted to understand the behavior of ferroresonance many unknowns regarding this phenomenon still exist, especially with regard to the initiation and the occurrence of different ferroresonance modes. Thus, this paper provides a comprehensive analysis of the signal qualities of three of the different modes of ferroresonance. This analysis was performed by processing voltage and current signals simulated using the ATP-EMTP software package. In the past, most research focused on the fundamental mode of ferroresonance [10], [11] but this paper also analyzes the subharmonic and chaotic modes of ferroresonance. This analysis should provide a better understanding of the differences between these three ferroresonance modes in terms of

\footnotetext{
* Manuscript received July 23, 2013; revised July 31, 2013.

Corresponding author. Tel.: +603-89287247; E-mail address: zaipatimah@uniten.edu.my.
} 
harmonic components and the total harmonic distortion. Such knowledge is essential when performing the tasks of identification, detection and classification of ferroresonance that are necessary if robust methods of ferroresonance mitigation are to be designed.

The paper is organized as follows: Section 2 describes the transmission network that is used when modeling the ferroresonance circuit. The modeling and simulation of the ferroresonance circuit is presented in Section 3. Section 4 discusses the spectral analysis of the ferroresonance simulated signals and the concluding remarks are presented in Section 5.

\section{Modeling and Simulation of the Ferroresonance Test Network}

This section presents the transmission network used for modeling the ferroresonance circuit. In Fig. 1, the single line diagram of the real system is considered. The ferroresonance occurs between the grading capacitance of the circuit breakers and the voltage transformer. The model has been adopted from [12] and represents the Dorsey high voltage direct current (HVDC) converter station in the Manitoba hydro system, Canada. The details of the system configuration and parameters are given in [12].

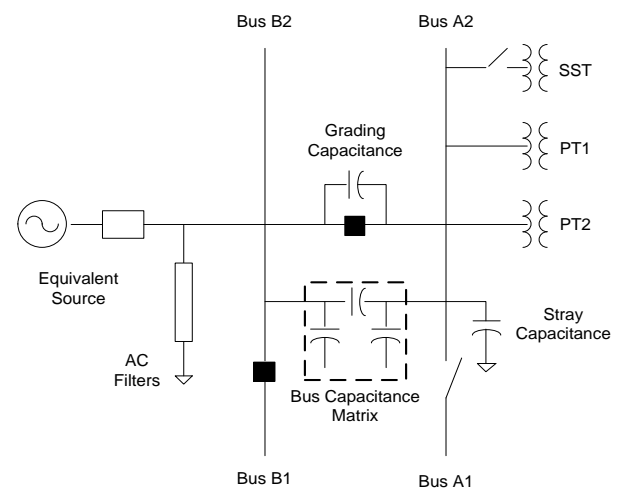

Fig. 1. System configuration for Dorsey HVDC converter station.

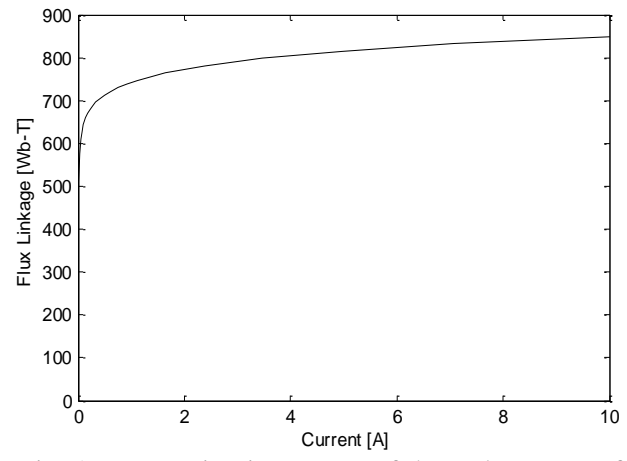

Fig. 2. Magnetization curve of the voltage transformer.

The modeling of the nonlinear transformer that exhibits ferroresonance is one of the most important aspects to consider when modeling the ferroresonance circuit. Fig. 2 depicts the magnetization curve that is used to model the nonlinearity of the voltage transformer. The model of the ferroresonance circuit in Fig. 1 is given in Fig. 3. This model was implemented using the alternative transient program-electro magnetic transient program (ATP-EMTP).

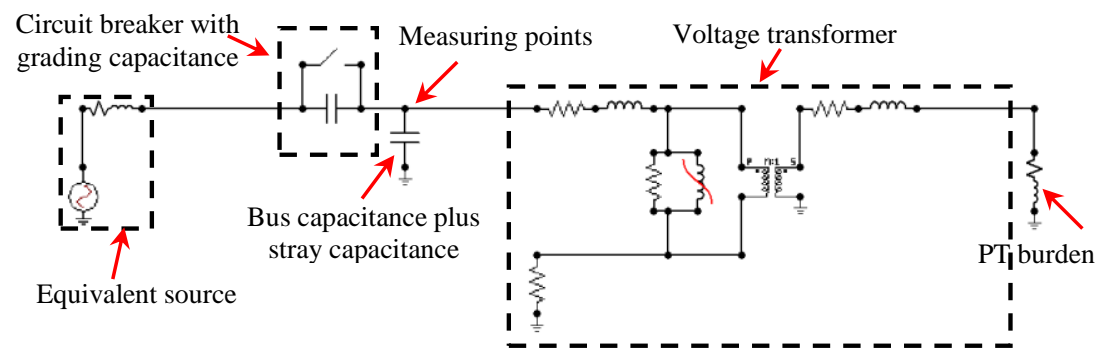

Fig. 3. EMTP model for Dorsey HVDC converter station.

Three modes of ferroresonance can be observed in the modelled circuit: the fundamental mode, the subharmonic mode and the chaotic mode. The voltage and current signals for each of these modes are presented in Fig. 4 through Fig. 6. These signals represent the response of the system when steady state operation is disturbed by the opening of the circuit breaker at $t=0.1 \mathrm{~s}$. Sustained ferroresonance has been achieved after a short transient period, the length of which was different for each of the modes of 
ferroresonance. A visual inspection of the shapes of the voltage signals reveals obvious differences that can be quantified through analysis in the spectral domain.

\section{Spectral Analysis of the Simulated Voltage Signals}

The Fourier transform provides a method for examining the level of distortion in the input signal. The most common applications of the Fourier transform are the analysis of linear time-invariant systems and spectral analysis. In this section, spectral analysis of the ferroresonance signals is performed by calculating the magnitude spectrum and the total harmonic distortion (THD).

The analysis of the signal is separated into the three different periods shown in Fig. 4. Time $t_{1}$ is the time when the circuit breaker switching operation occurred. Time $t_{2}$ is defined as the time when the system enters the sustained ferroresonance. The signals in each period can be described as: Normal during Period $1 t<t_{1}$, transient during Period $2 t>t_{1}$ and $t<t_{2}$ and sustained ferroresonance during Period $3 t>t_{2}$.

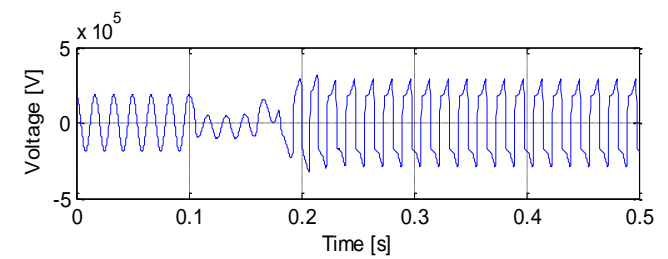

(a)

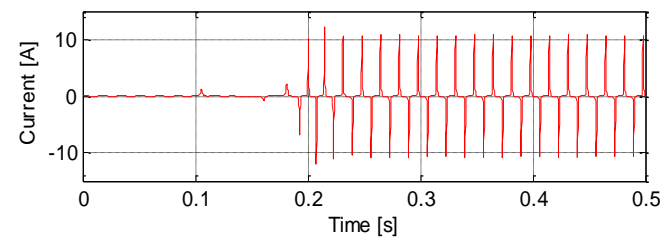

(b)

Fig. 4. Fundamental ferroresonance mode (a) voltage signal and (b) current signal.

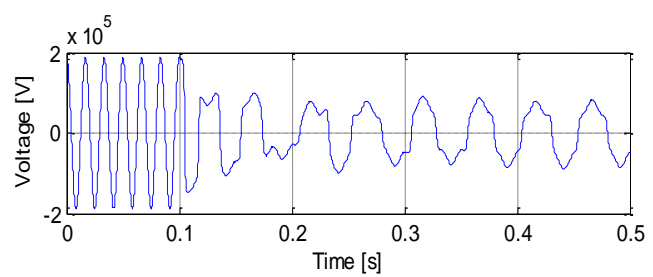

(a)

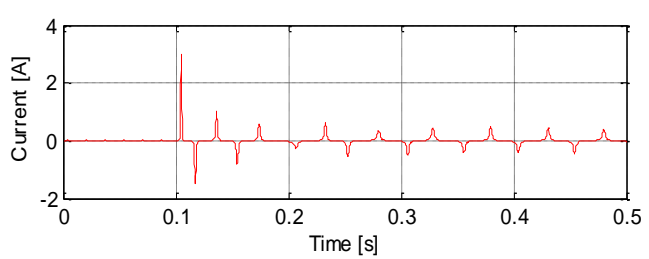

(b)

Fig. 5. Subharmonic ferroresonance mode (a) voltage signal and (b) current signal.

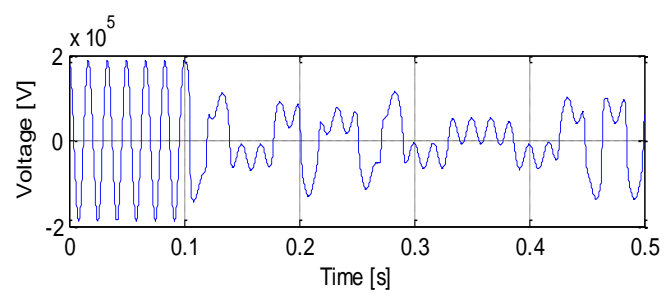

(a)

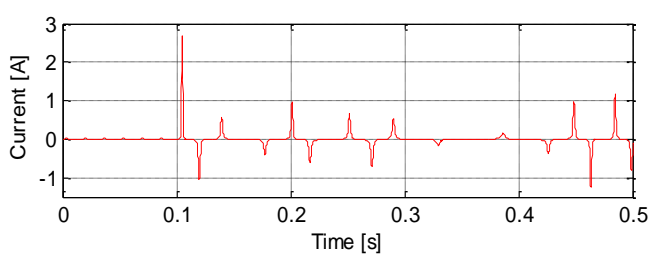

(b)

Fig. 6. Chaotic ferroresonance mode (a) voltage signal and (b) current signal.

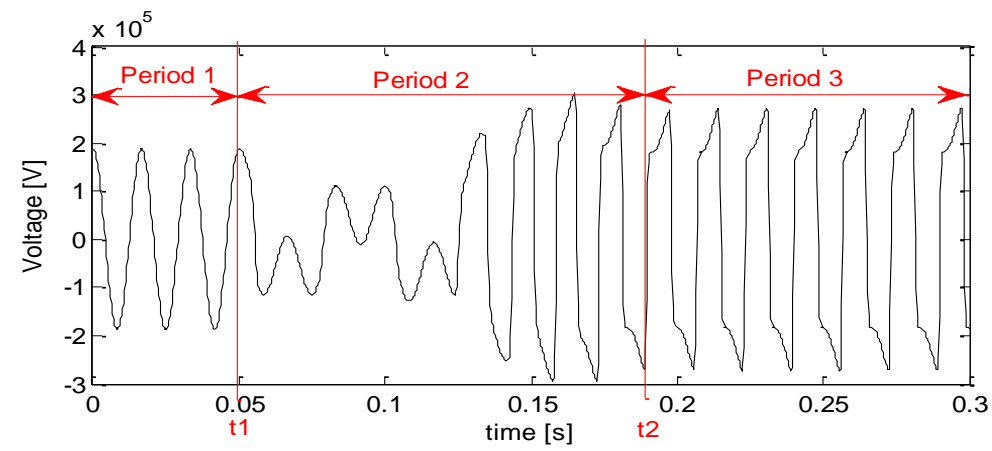

Fig. 7. Definition of periods 1,2 and 3 of the processed signal. 


\subsection{Magnitude spectrum}

The discrete Fourier transform (DFT) is a mathematical procedure that is used to determine the harmonic or frequency components of a discrete signal. The DFT is defined as follows:

$$
X[k]=\sum_{n=0}^{N-1} x[n] e^{-j \frac{2 \pi k n}{N}}
$$

where $X[k]$ is the complex value of the discrete sequence in the frequency domain, $N$ is the number of samples of the input sequence and the number of frequency points in the DFT output, $x[n]$ is the sequence of input samples in the time domain, $n$ is the index of the input samples in the time domain and $k$ is the index of the DFT output in the frequency domain that is defined as:

$$
k=1,2,3 \mathrm{~K} N-1
$$

The magnitude spectrum for each of modes of ferroresonance has been obtained by applying the DFT to a portion of Period 3 of the ferroresonance signals. The voltage and current signals for each ferroresonance mode are shown alongside the corresponding magnitude spectrums in Fig. 8 through Fig. 13.

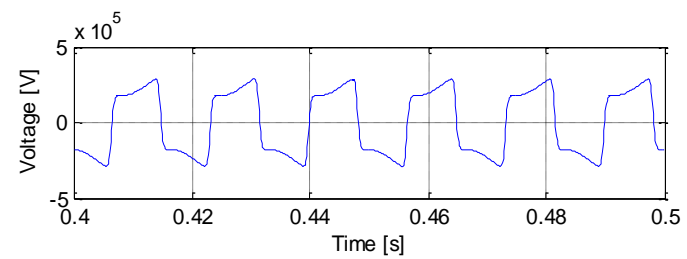

(a)

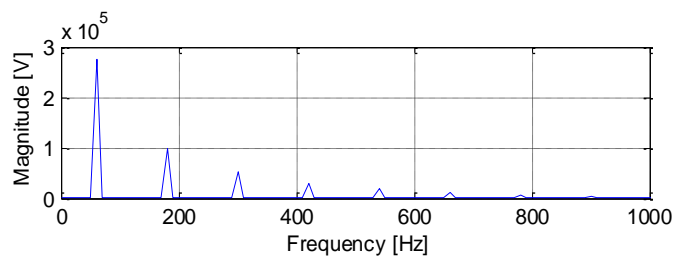

(b)

Fig. 8. Sustained fundamental ferroresonance mode (a) voltage signal and (b) voltage magnitude spectrum.

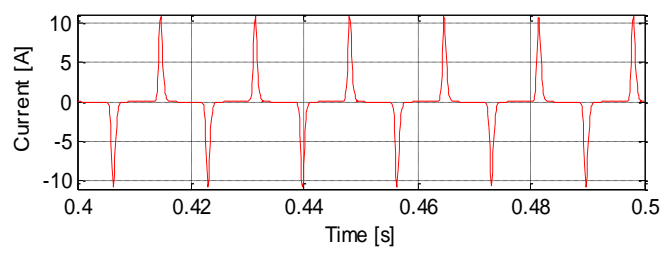

(a)

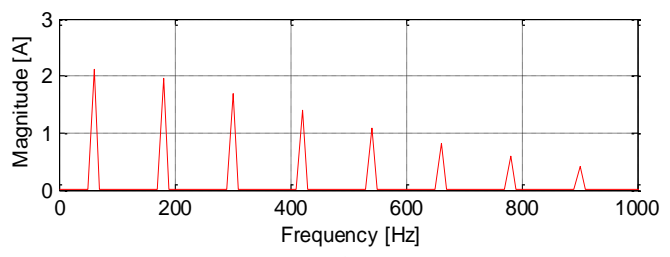

(b)

Fig. 9. Sustained fundamental ferroresonance mode (a) current signal and (b) current magnitude spectrum

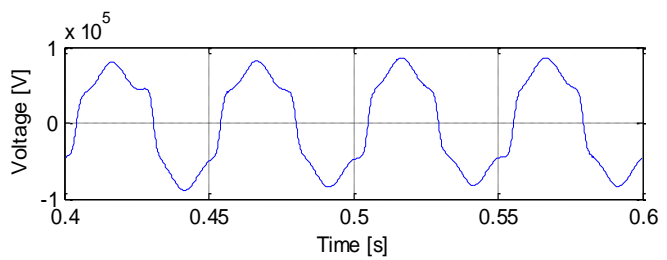

(a)

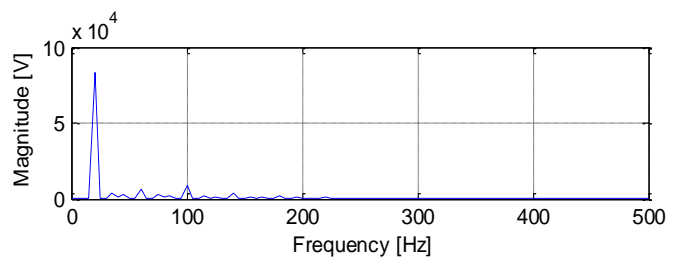

(b)

Fig. 10. Sustained subharmonic ferroresonance mode (a) voltage signal and (b) voltage magnitude spectrum.

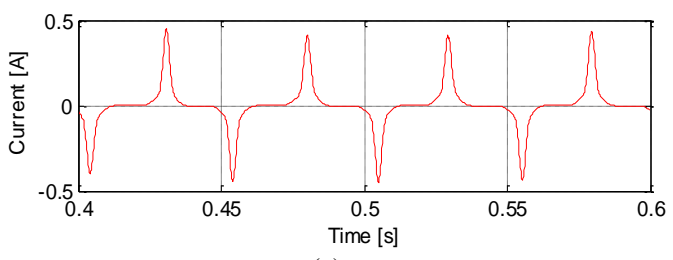

(a)

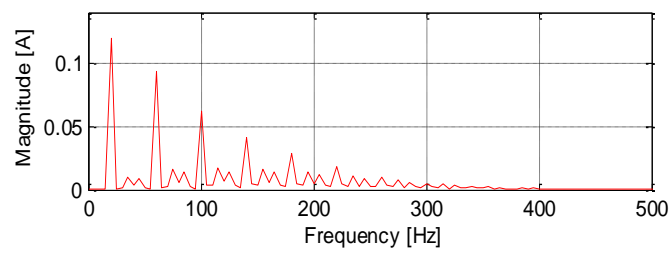

(b)

Fig. 11. Sustained subharmonic ferroresonance mode (a) current signal and (b) current magnitude spectrum. 


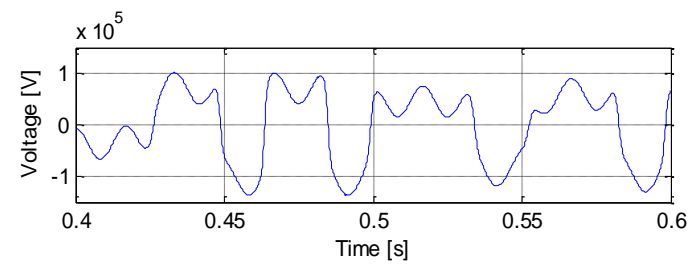

(a)

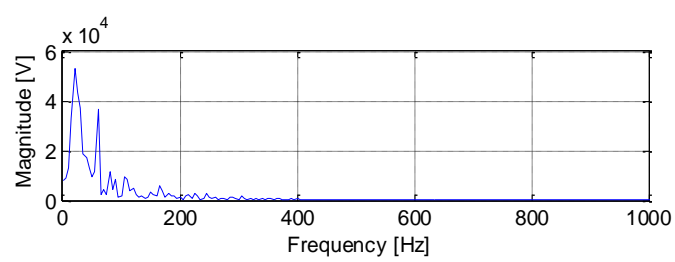

(b)

Fig. 12. Sustained chaotic ferroresonance mode (a) voltage signal and (b) voltage magnitude spectrum.

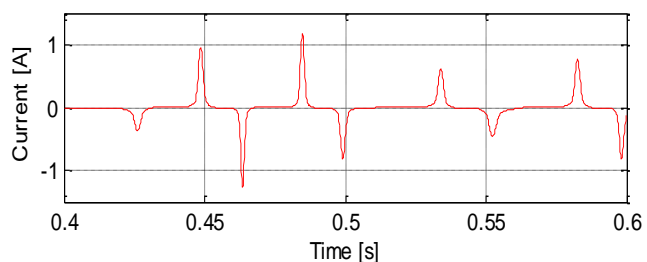

(a)

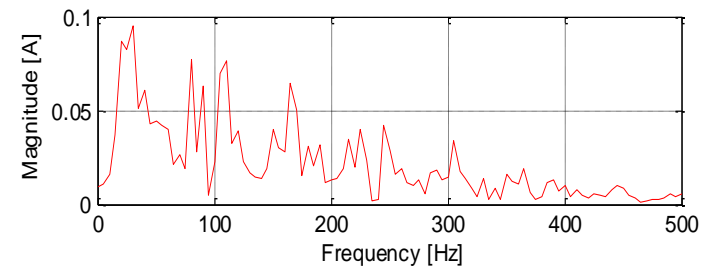

(b)

Fig. 13. Sustained chaotic ferroresonance mode (a) current signal and (b) current magnitude spectrum.

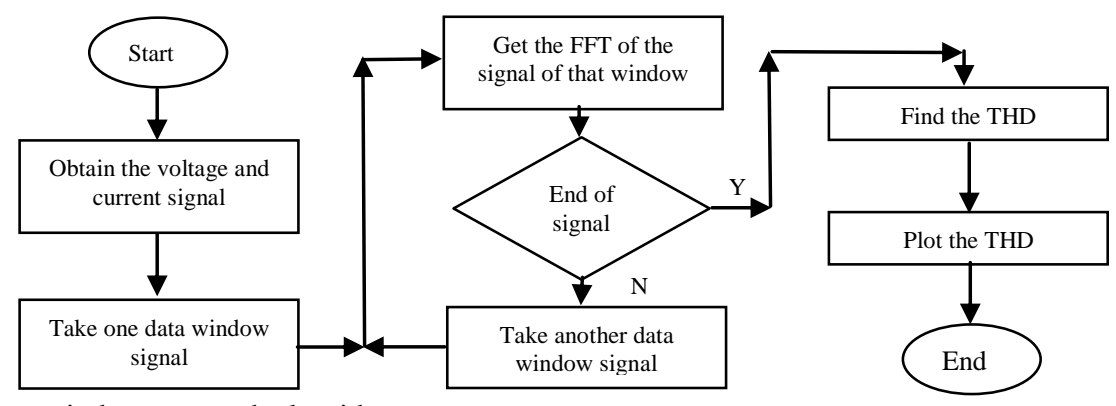

Fig. 14. THD data window approach algorithm.

It can be observed that during the sustained ferroresonance the voltage and current of three modes of ferroresonance have different magnitude spectrums. The fundamental mode has a spectrum that is an odd multiple of the system frequency. The subharmonic mode contains a sub-frequency $(1 / n)$ of the system frequency. The chaotic mode lacks discrete bands of frequency content; instead the spectrum is broadband and varies continuously across the frequency range.

\subsection{Derivation of the total harmonic distortion}

A harmonic of a signal is defined as a frequency component of the signal that is an integer multiple of the fundamental frequency. For example, given a $60 \mathrm{~Hz}$ fundamental signal, the 2nd, 3rd, and 4th harmonic components will be at $120 \mathrm{~Hz}, 180 \mathrm{~Hz}$ and $240 \mathrm{~Hz}$, respectively. Harmonics have always been present in power systems. However, the harmonic components were so small that their effects on systems were negligible and the voltage and current signals could be considered as ideal sine waves with zero harmonic content. However, when a nonlinear load draws current that is not perfectly sinusoidal, the voltage signal will be distorted. Harmonic distortion can have harmful effects on electrical equipment. Unwanted distortion can increase the current in power systems, which will result in higher temperatures in neutral conductors and distribution transformers.

Total harmonic distortion (THD) is used to compare the summation of all of the harmonic components of the voltage or current waveform with the fundamental. The THD of a voltage signal, $V$, is calculated by using the following equation:

$$
T H D=\frac{\sqrt{\sum\left|V_{2}^{2}+V_{3}^{2}+V_{4}^{2}+\mathrm{L}+V_{N}^{2}\right|}}{\left|V_{1}\right|} \times 100 \%
$$


where $V_{1}$ is the fundamental or first harmonic and $V_{2}, V_{3} \ldots V_{N}$ are the $2 \mathrm{nd}$, 3rd, and $N$ th harmonics, respectively.

The total harmonic distortion was calculated for the three different modes of ferroresonance to evaluate the deviation of the ferroresonance mode signals from a pure sinusoidal signal. This analysis was conducted using a sliding data window. The harmonic components in each data window are obtained using the Fourier Transform. The THD is then calculated for this particular data window. The process is repeated after sliding the window one sample forward until the end of the signal is reached. This process is summarized in Fig. 14. The data window size that is used in this analysis is $2 T_{0}$, where $T_{0}$ is defined as:

$$
T_{0}=\frac{1}{60}=16.667 \mathrm{~ms}=1 \text { period }
$$

The number of samples per $T_{0}$ is 128 samples, so each data window contains 256 samples. This process allows the harmonic waveforms depicted in Fig. 15 (a) for each of the modes of ferroresonance to be determined.

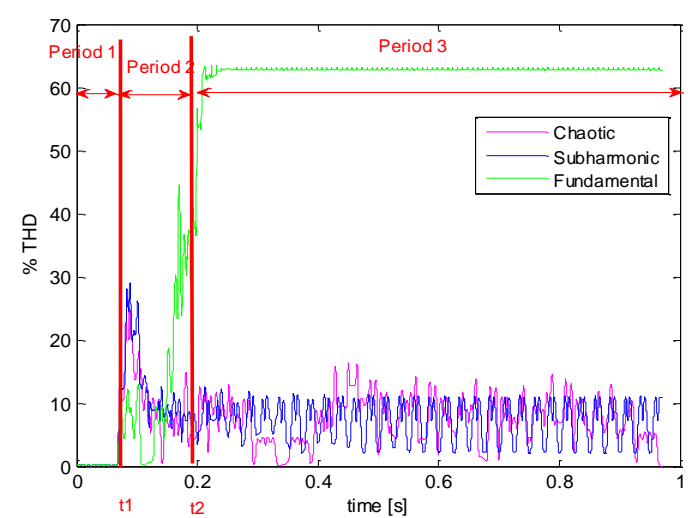

(a)

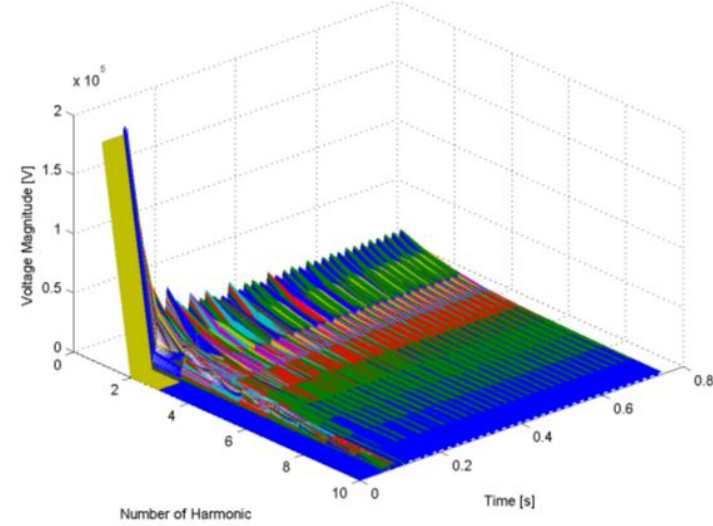

(c)

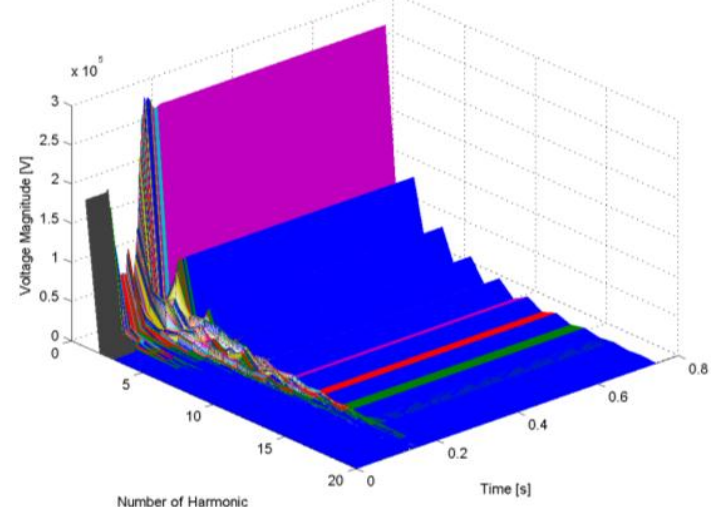

(b)

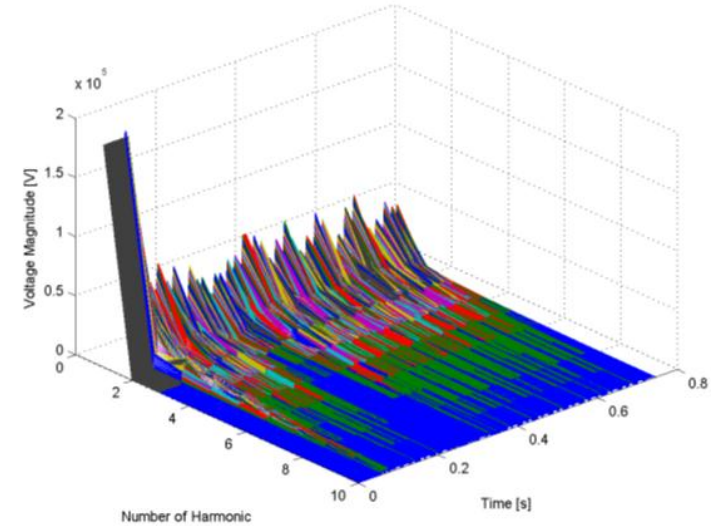

(d)

Fig. 15. Total harmonic distortion of the three modes (a), harmonic content of the three modes: (b) fundamental mode ferroresonance, (c) subharmonic mode ferroresonance, (d) chaotic mode ferroresonance that are extracted using the DFT and a sliding data window.

The harmonic content in the fundamental ferroresonance mode during the sustained period (Period 3) is constant in nature. However, for the subharmonic and chaotic modes of ferroresonance, the harmonic content changes throughout Period 3. The harmonic content of the subharmonic mode oscillates whilst for the chaotic mode it varies chaotically. Fig. 15 (b) through (d) depict the harmonic contents of the three 
ferroresonance mode. Further analysis of these signals is necessary in order to extract the distinguishing features that would allow the identification and classification of ferroresonance modes in future.

\section{Conclusion}

Feature extraction is necessary if robust methods are to be designed for the classification and mitigation of ferroresonance. Spectral analysis of three different modes of ferroresonance has shown that each mode has different characteristics that could be used as the basis for feature extraction and for distinguishing between each mode. The THD of the fundamental mode is a distinctive feature but the THD of the subharmonic and chaotic mode will require further analysis before it can be used.

\section{References}

[1] Ferracci P. Ferroresonance. Group Schneider: Cahier Technique no 190, 1998.

[2] Iravani MR, Chaudhary AKS., Giesbrecht WJ, et al. Modeling and analysis guidelines for slow transients. III. The study of ferroresonance. IEEE Transactions on Power Delivery, 2000; 15(1):255-265.

[3] Charalambous C, Wang ZD, C, lie J, Osborne M, Jarman P. Validation of a power transformer model for ferroresonance with system tests on a $400 \mathrm{kV}$ circuit. Presented at: International Conference on Power Systems Transients, 2007.

[4] Moses PS, Masoum MAS. Experimental and simulation analysis of ferroresonance in single-phase transformers considering magnetic hysteresis effects. Presented at: IEEE Power and Energy Society General Meeting, 2010.

[5] Huang S-J, Hsieh C-H. Relation analysis for ferroresonance of bus potential transformer and circuit breaker grading capacitance. International Journal of Electrical Power \& Energy Systems, 2013; 51:61-70.

[6] Lacerda Ribas JC, Lourenco EM, Vianei Leite J, Batistela NJ. Modeling ferroresonance phenomena with a flux-current JilesAtherton hysteresis approach. IEEE Transactions on Magnetics, 2013; 49(5):1797-1800.

[7] Ben Amar F, Dhifaoui R. Study of the periodic ferroresonance in the electrical power networks by bifurcation diagrams. International Journal of Electrical Power \& Energy Systems, 2011; 33(1):61-85.

[8] Radmanesh H, Gharehpetian GB. Ferroresonance suppression in power transformers using chaos theory. International Journal of Electrical Power \& Energy Systems, 2013; 45(1):1-9.

[9] Mokryani G, Haghifam M-R, Latafat H, Aliparast P, Abdolahi A. Wavelet based Kernel fisher classifier for ferroresonance identification. In: Proc. of 15th International Conference on Intelligent System Applications to Power Systems, 2009:1-6.

[10] Akinci TC, Ekren N, Seker S, Yildirim S. Continuous wavelet transform for ferroresonance phenomena in electric power systems. International Journal of Electrical Power \& Energy Systems, 2013; 44(1):403-409.

[11] Seker S, Akinci T, Taskin S. Spectral and statistical analysis for ferroresonance phenomenon in electric power systems. Electrical Engineering, 2012; 94(2):117-124.

[12] Jacobson DAN, Mazur RW. Mitigating potential transformer ferroresonance in a $230 \mathrm{kV}$ converter station. Presented at: Transmission and Distribution Conference, 1996. 